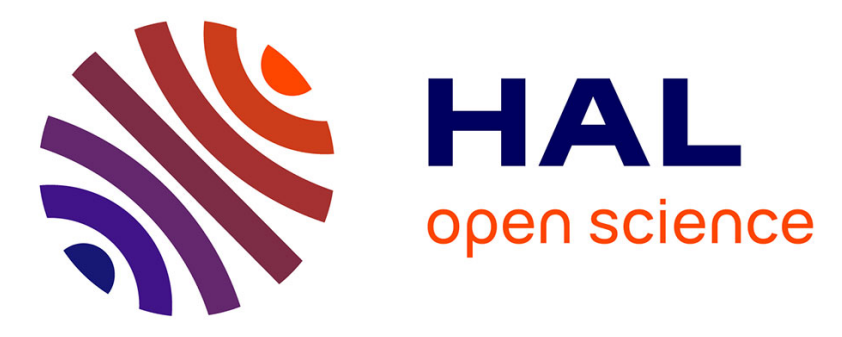

\title{
Tailoring and imaging the plasmonic local density of states in crystalline nanoprisms
}

Sviatlana Viarbitskaya, Alexandre Teulle, Renaud Marty, Jadab Sharma, Christian Girard, Arnaud Arbouet, Erik Dujardin

\section{- To cite this version:}

Sviatlana Viarbitskaya, Alexandre Teulle, Renaud Marty, Jadab Sharma, Christian Girard, et al.. Tailoring and imaging the plasmonic local density of states in crystalline nanoprisms. Nature Materials, 2013, 12 (5), pp.426-432. 10.1038/nmat3581 . hal-01798016

\section{HAL Id: hal-01798016 https://hal.science/hal-01798016}

Submitted on 19 Feb 2021

HAL is a multi-disciplinary open access archive for the deposit and dissemination of scientific research documents, whether they are published or not. The documents may come from teaching and research institutions in France or abroad, or from public or private research centers.
L'archive ouverte pluridisciplinaire HAL, est destinée au dépôt et à la diffusion de documents scientifiques de niveau recherche, publiés ou non, émanant des établissements d'enseignement et de recherche français ou étrangers, des laboratoires publics ou privés. 
Tailoring AND imaging the Plasmonic local Density of States In Crystalline NANOPRISMS

Sviatlana Viarbitskaya, Alexandre Teulle, Renaud Marty, Jadab Sharma, Christian Girard, Arnaud Arbouet* and Erik Dujardin*

CEMES CNRS UPR 8011 and Université de Toulouse, 29 rue Jeanne Marvig, BP 94347, 31055 Toulouse cedex 4, France.

e-mail: arbouet@cemes.fr, dujardin@cemes.fr

\begin{abstract}
Surface plasmon (SP) technologies exploit the spectral and spatial properties of collective electronic oscillations in noble metals placed in an incident optical field. Yet the SP local density of states (LDOS), that rules the energy transducing phenomena between the SP and the electromagnetic field, is much less exploited. Here, we use twophoton luminescence (TPL) microscopy to reveal the SP-LDOS in thin single crystalline triangular gold nanoprisms produced by a quantitative one-pot synthesis at room temperature. Variations of the polarization and the wavelength of the incident light redistribute the TPL intensity into $2 \mathrm{D}$ plasmonic resonator patterns that are faithfully reproduced by theoretical simulations. We demonstrate that experimental TPL maps can be considered as the convolution of the SP-LDOS with the diffraction-limited Gaussian light beam. Finally, the SP modal distribution is tuned by the spatial coupling of nanoprisms, thus allowing a new modal design of plasmonic information processing devices.
\end{abstract}


The physics and engineering of surface plasmons (SP) in metal films have opened the prospects of optical information processing beyond the diffraction limit. ${ }^{1-3}$ Plasmonic circuitry at the micrometer-scale has been successfully demonstrated ${ }^{4}$ and finds technologicallyrelevant applications in telecommunications. ${ }^{5,6}$ The evanescent extension of the SP modes into the surrounding environment creates or modulates electromagnetic modes near the metallic structures. The optical near-field generated by these modes has been probed by a number of near- and far-field optical techniques such as leakage radiation microscopy, ${ }^{7}$ Scanning Near-Field Optical Microscopy (SNOM), ${ }^{8,9}$ enhanced fluorescence ${ }^{10,11}$ or Raman microscopy. ${ }^{12}$ Furthermore, apertured SNOM probes the spectral and spatial distribution of the local density of these photonic states (Ph-LDOS) so close above a plasmonic surface at the tip position that the features resembled SP modes themselves. ${ }^{13}$ Two-Photon Luminescence (TPL) intensity has been correlated to SP resonances ${ }^{14}$ and was shown to provide quantitative field enhancement measurements ${ }^{15}$ and diffraction-limited plasmon mode maps. ${ }^{16,17}$ The TPL intensity maxima could even be located with sub-diffraction resolution by photon localization analysis. ${ }^{18}$ However, to date, only few techniques can image the local density of the plasmonic states (SP-LDOS) inside the metallic nanostructures. Cathodoluminescence (CL), ${ }^{19}$ photoelectron emission microscopy (PEEM) $)^{20,21}$ and, especially, electron energy-loss spectroscopy (EELS) and energy filtered microscopy have revealed unique SP modal maps of nanometric ${ }^{22,23}$ and mesoscopic ${ }^{24}$ colloidal platelets. Noteworthy, these techniques only probe some components of the LDOS. In EELS, the intensity map can be related to the integral over the electron path of the Z-component of the LDOS. ${ }^{25}$ Similarly, in CL, the radiated power is proportional to the partial LDOS along the direction of the transient dipole at the impact point. ${ }^{19}$ The design of SP mode-based information processing would therefore benefit from a technique able to map the full SP-LDOS, which underpins energy localization and transfer phenomena by SP modes. ${ }^{26-28}$ Concomitantly, the ultimate control of the eigenmodes of these 2D electron gases could provide an ambitious downscaling technology to the nanometerscale. ${ }^{28,29}$ To this end, the material engineering of plasmonic systems must provide a precise geometrical design and an improved single crystallinity of the metal nanostructures, for which colloidal chemistry is much better performing than standard lithography and its associated metal film processing. ${ }^{30-33}$

Here, we show that TPL microscopy is suited to directly visualize SP-LDOS inside ultrathin planar gold microcrystals. 20-nm thick gold triangular, truncated triangular and hexagonal nanoprisms of typical lateral size ranging from 0.5 to $1.0 \mu \mathrm{m}$ are synthesized by an original and quantitative method. TPL maps recorded on individual triangular nanoprisms exhibit a 
highly localized intensity distribution, which is dependent on both incident polarization and wavelength. A novel formalism that faithfully reproduces the experimental patterns demonstrates that the TPL intensity is directly proportional to the square of the in-plane SPLDOS in the high quality 2D resonators. Moreover, we observe that the modal distribution can be tuned by the spatial coupling of nanoprisms. This opens a new route towards plasmonic information processing classical and quantum devices, which is illustrated by a proposed implementation of a plasmonic modal logic gate.

Single crystalline Au or Ag nanostructures produced by colloidal chemistry sustain high quality factor plasmonic modes thanks to their low dissipation. ${ }^{31}$ The extension of single crystalline growth to larger sizes enables the investigation of the spatial distribution of electric fields and SP modes by simple optical microscopy on micrometric objects such as pentatwinned Au or Ag nanowires. ${ }^{30,34,35}$ Yet atomically flat crystalline platelets are better suited for the observation of the two-dimensional (2D) extension of SP modes. We have produced Au nanoprisms with micrometric lateral sizes by a new one-pot method based on the direct reduction of Au precursors by polyvinylpyrrolidone (PVP) in alkaline conditions at room temperature (See Methods and Supplementary Information, SI). Figure 1a shows that the sample is composed of 0.5-1.0 $\mu \mathrm{m}$ equilateral triangular platelets with various degrees of truncation reaching the symmetrical hexagonal morphology. For TPL experiments, the nanoprisms are dispersed onto cleaned conductive glass substrates. The adsorbed PVP is removed by washing with deionized water and a short oxygen plasma etching. Specific nanoprisms that undergo TPL investigations are characterized before and after laser illumination by SEM and their height is measured by atomic force microscopy (AFM). This procedure ensures that all TPL data have been acquired without laser-induced modification of the nanoprisms and provides the accurate nanoprism size and morphology for TPL map simulations.

Figure $1 \mathrm{~b}$ is a scheme of the TPL experiments performed on a home-built optical microscope illuminated with a Ti:Sapphire femtosecond laser. The polarized excitation beam is tightly focused in a $300 \mathrm{~nm} \mathrm{fwhm}^{36}$ spot and the TPL intensity is collected in epi-collection geometry as the nanoprisms sample is raster scanned (See Methods). The TPL images of individual nanoprisms are matched with their SEM micrographs allowing an unambiguous identification of each object. Figures 1c and 1d show the SEM image and unprocessed TPL intensity map of an equilateral triangular nanoprism of $920 \mathrm{~nm}$ in side length and $20 \mathrm{~nm}$ in height illuminated with a linearly polarized light $(\lambda=700 \mathrm{~nm})$. The experimental map presents a well-resolved 
pattern composed of three circular spots of different intensities located at the apexes of the nanoprisms. A weaker signal along the triangular edges is also visible but no measurable luminescence emerges from the center of the prism. For a better comparison with simulated images, the raw TPL maps are smoothed by applying a Gaussian interpolation filter of minimal diameter that does not alter the informational content of the images (See SI).

In order to investigate the origin of this sub-particle pattern, we have recorded series of TPL maps for different in-plane polarizations of the linearly polarized incident light as illustrated in Figures 2a-f for the triangular prism with lateral size of $790 \mathrm{~nm}$ shown in Figure $2 \mathrm{~g}$ and illuminated at $\lambda=800 \mathrm{~nm}$. For an incident polarization parallel to the lower side of the prism (Fig. 2d), a single bright TPL spot is observed on the distal apex with two very weak spots on the proximal apexes. The observed patterns are orthogonal to the near-field pattern predicted for smaller prisms (ca. $100 \mathrm{~nm}$ ) and observed by $\mathrm{SNOM}^{9}$ or PEEM, ${ }^{37}$ which are composed of two bright spots along the edge parallel to the incident polarization. Upon rotating the polarization anti-clockwise (Figs. 2d-a), the lower right apex appears brighter with an equal intensity recorded on both apexes when the incident polarization is aligned with the bisector. Similarly, a bright spot emerges on the lower left corner with the clockwise rotation of the polarization (Fig. 2d-f). A $180^{\circ}$ turn of the incident polarization thus results in the successive illumination of each three apexes and in a periodical variation of the TPL intensity integrated in an area equivalent to the minimal objective focal size (ca. $300 \mathrm{~nm}) .^{38}$ The remarkable evolution of these TPL patterns can be better understood by an accurate theoretical description. TPL is an incoherent non-linear emission process, in which the absorption of two photons is followed by a complex non-radiative decay and finally by a radiative recombination. In first approximation, the TPL intensity is proportional to the fourth power of the near-field amplitude of the electric field. ${ }^{15-18,39}$ We have recently developed a TPL simulation tool that faithfully accounts for the Gaussian shape of the exciting field (see Methods and SI). ${ }^{40}$ Figures $2 \mathrm{j}$-o show the calculated maps corresponding to Figures 2a-f for a simulated beam waist of $270 \mathrm{~nm} .{ }^{36}$ The agreement between simulated and experimental maps is excellent, not only with respect to the $2 \mathrm{D}$ distributions of intensity, which displays the same spot patterns with the matching intensity ratio, but also with respect to the linear polarization dependency. This suggests that the TPL maps result from the projection of $\mathrm{C}_{3 \mathrm{v}}$ symmetrical plasmonic modes borne inside the ultrathin prisms with the incident linearly polarized field. The direct imaging of the 2D plasmonic resonator modes by TPL is further supported by monitoring the variation of the intensity pattern as the excitation wavelength is tuned. The TPL maps of a triangular prism of lateral size $780 \mathrm{~nm}$ are shown for a series of incident 
wavelength at fixed polarization in Figure 3, along with the corresponding simulated images. While the excitation at $700 \mathrm{~nm}$ with a polarization parallel to one edge yields a bright spot on the distal corner (Fig. 3a), the increase of the excitation wavelength to 750 and $800 \mathrm{~nm}$ spreads the intensity more evenly with a gradual increase of the spot located on the neighbouring apex (Figs. 3b and 3c). Finally, when excited at $850 \mathrm{~nm}$, the TPL intensity is mostly distributed along the proximal edge with a maximum on the third apex (Fig. 3d). The general patterns and rotation of the relative spot intensities are fully accounted for in the simulated maps (Fig. 3e-h). The triangular prisms, that are large and, importantly, in-plane single crystals, behave like 2D resonators in which higher-order SP eigenmodes emerge that can be probed by diffraction-limited TPL microscopy. ${ }^{24,31}$

To better describe these observations, we remind that the relaxation of classical or quantum emitters placed in the vicinity of a metallic nanostructure is governed by the entire set of available electromagnetic eigenmodes. Similarly, we can consider the dipole induced by the excitation beam inside the metallic structure itself. In Supplementary Information, we demonstrate that the power radiated by this induced dipole is related to the full SP-LDOS, inside the structure, projected on the dipole direction, rather than to a particular eigenmode. To probe the entire planar component of the SP-LDOS, $\rho_{\|}$, we consider a circularly polarized incident light of wavelength $\lambda_{0}=2 \pi / \omega_{0}$, impinging at $\mathrm{R}_{0}$ on a plasmonic structure. The intensity of the linear emission, generated by the induced dipole at a point $r$, is proportional to the square of the local electric field magnitude, $\mathrm{E}\left(\mathrm{r}, \mathrm{R}_{0}, \omega_{0}\right)$. On another hand, the emission intensity is also directly linked to the product of the excitation field intensity, $\left|\mathbf{E}_{\mathbf{0}}\left(\mathbf{r}, \mathbf{R}_{\mathbf{0}}, \omega_{0}\right)\right|^{2}$ by $\rho_{\|}$(See SI).$^{41}$ Consequently, the local field intensity can be written as:

$$
\left|\boldsymbol{E}\left(\boldsymbol{r}, \boldsymbol{R}_{\mathbf{0}}, \omega_{0}\right)\right|^{2} \propto \omega_{0}^{2}\left|\boldsymbol{E}_{\mathbf{0}}\left(\boldsymbol{r}, \boldsymbol{R}_{\mathbf{0}}, \omega_{0}\right)\right|^{2} \rho_{\|}\left(\boldsymbol{r}, \omega_{0}\right)
$$

If one integrates (1) over the entire volume, $\mathrm{V}$, of the metallic nanostructure, the TPL intensity generated by a circularly polarized light excitation follows the product of the fourth power of the exciting field by the SP-LDOS squared:

$$
I_{T P L}\left(\boldsymbol{R}_{\mathbf{0}}, \omega_{0}\right) \propto \omega_{0}^{4} \int_{V}\left|\boldsymbol{E}_{\mathbf{0}}\left(\boldsymbol{r}, \boldsymbol{R}_{\mathbf{0}}, \omega_{0}\right)\right|^{4} \rho_{\|}^{2}\left(\boldsymbol{r}, \omega_{0}\right) d \boldsymbol{r}
$$

If the incident field is linearly polarized (Figs. 2 and 3), the TPL signal is proportional to the squared projection of the partial SP-LDOS along the polarization direction. Figure 4a shows the experimental evidence that the circularly polarized excitation of the nanoprisms indeed yields a symmetrical pattern comprising three identical spots located at each apex. Alternatively, the complete TPL intensity can be reconstructed by summing the maps obtained for two orthogonal incident polarizations as shown experimentally in Figure 4b and 
faithfully reproduced by corresponding numerical calculations with a beam waist of $270 \mathrm{~nm}$ in Figure 4c. Thus, TPL microscopy probes the plasmonic states inside the metal when the light impinges strictly on the metallic structure, whereas SNOM, for example, probes the photonic states that protrude further outside the metal and EELS-EFTEM of thin 2D structures has been shown to essentially follow the z-component of the SP-LDOS (See SI). ${ }^{13,25}$ Interestingly, since the beam waist model embedded in our numerical approach has proven to accurately reproduce all experimental observations, which are obviously diffraction-limited, our simulation tool can be used to extrapolate the TPL signal of an infinitely narrow probing beam (See SI). When the beam waist is theoretically contracted towards zero, equation (2) becomes:

$$
I_{T P L}^{0}\left(\boldsymbol{R}_{\mathbf{0}}, \omega_{0}\right) \propto \omega_{0}^{4} \rho_{\|}^{2}\left(\boldsymbol{R}_{\mathbf{0}}, \omega_{0}\right)
$$

Figure 4d, which corresponds to the TPL map of Fig. 4b extrapolated at $60-\mathrm{nm}$ beam waist, provides an image of the squared SP-LDOS of the prism. Secondary spots, which are not resolved experimentally in Fig. 4b, appear clearly confined along each prism side, which is a manifestation of the edge modes emerging specifically in crystalline structures, as observed by EELS in Au nanoplatelets ${ }^{24}$ and leakage radiation microscopy in long Ag penta-twinned nanorods. ${ }^{35}$ Noteworthy, the four spots on each side are equidistant suggesting that the main contribution to the TPL signal, at the considered energy, originates from a third order mode of the 2D triangular resonator. Hence, TPL microscopy straightforwardly provides maps of the in-plane SP-LDOS inside our highly crystalline and large sized 2-dimensional prisms convoluted with the finite-sized excitation beam profile and reveals the higher order SP features. Our findings further show that the TPL patterns can be manipulated by the projection of the SP-LDOS onto the polarization of the incident light.

Exploiting the low level of dissipation observed in our structures, we propose the novel design of plasmonic modal logic gates by engineering the rich 2D SP-LDOS of thin prisms to produce delocalized modes at desired energies and to construct a planar plasmonic information processing architecture. This holistic approach to plasmonic circuitry complements the recent analogic implementation using phase-controlled plasmonic interferential logic gates in coupled nanowires. ${ }^{28,42}$

As a first step in this direction, we consider the influence of the coupling by proximity of individual prisms on the 2D SP-LDOS pattern. In Figure 5, two quasi-identical truncated triangular prisms (A) and (B) lie at $50 \mathrm{~nm}$ from each other in a bowtie-like configuration (Fig. 5b). TPL maps are recorded for two different linear polarizations, parallel (Fig. 5a) and perpendicular (Fig. $5 \mathrm{c}$ ) to the dimer longitudinal axis $(\lambda=800 \mathrm{~nm})$. Figure 5a shows a rather 
homogenous distribution of the TPL intensity over the entire dimer, while Figure 5c displays an intense spot confined in the gap separating the two prisms. The modification of the SPLDOS by the close proximity of the two structures is evidenced by calculating the TPL maps for the parallel polarization for the entire (AB) dimer (Fig. 5d) or for the prisms (A) and (B) alone (Fig. 5e and $5 f$ respectively). The simple superposition of the individual TPL responses $(\mathrm{A})+(\mathrm{B})$, corresponding to non-interacting prisms displays an intense spot in the gap with a weaker pattern at the opposite edge of each prism (Fig. 5g). This does not match the experimental TPL map in Figure 5a, which is much better accounted for by the computation of the full dimer (Fig. 5d), implying that the SP-LDOS of the isolated prisms is reshaped in the dimer. These observations suggest that the shaping and coupling of colloidal structures foster the engineering of the spatial distribution of the SP-LDOS, $\rho_{\|}$. While the initial design sets the complex SP-LDOS pattern of assembled colloids, such planar devices can be easily exploited by projecting the SP-LDOS on a chosen linear polarization direction, which defines the area from which a strong TPL signal is emitted. This is illustrated in Figure 6, where the dimeric SP-LDOS (Fig. 6a) is explored in the TPL maps calculated for an excitation at $800 \mathrm{~nm}$ and a rotating linear polarization of the incident light with a realistic beam waist of $270 \mathrm{~nm}$. One can notice that the intensity in the positions $\mathrm{O}_{1}$ and $\mathrm{O}_{2}$ varies significantly when the excitation polarization varies between $5^{\circ}$ and $120^{\circ}$. Remarkably, the correlation of the TPL intensity in positions $\left(\mathrm{I}_{1}, \mathrm{I}_{2}\right)$ and $\left(\mathrm{O}_{1}, \mathrm{O}_{2}\right)$, as the polarization is rotated, suggests that such large coupled structures could be a building block of larger plasmonic information processing architectures. Indeed, a gedanken experiment consists in tightly focusing two laser spots of identical wavelength and power but independently chosen linear polarizations on positions $\mathrm{I}_{1}$ and $\mathrm{I}_{2}$ and in reading the TPL intensity in similar areas in $\mathrm{O}_{1}$ or $\mathrm{O}_{2}$ (Fig. 6g). The excitation polarization states, for example $5^{\circ}$ and $85^{\circ}$, would encode the ' 0 ' and ' 1 ' inputs respectively. The two pulsed inputs on $I_{1}$ and $I_{2}$ would be slightly delayed from each other to obviate interferences, while the output intensities are integrated during the entire illumination time span. In these conditions, the read-out intensity in $\mathrm{O}_{1}$ and $\mathrm{O}_{2}$ can be visualized by the summation of the maps in Figures $6 \mathrm{~b}$ and/or 6e, weighted by the TPL intensity at the corresponding input for the considered polarization and are represented as the background of Figures 6g-j. For a binary coding, the TPL intensity exceeding a threshold (uniform white color in the intense areas of Fig. 6g-j) encodes a '1' and any lower intensity is considered as a '0' output. In our example, the dimer would yield the binary response summarized in Figure $6 \mathrm{k}$. The Boolean logic relationship between $\mathrm{O}_{1}$ and $\left(\mathrm{I}_{1}, \mathrm{I}_{2}\right)$ therefore corresponds to a 
NAND gate, one of the fundamental gates, and $\mathrm{O}_{2}$ is the direct copy of $\mathrm{I}_{1}$, an important function that conveys an input to the next logic gate in complex 2D logic architectures. ${ }^{43}$

To conclude, we have produced large crystalline Au nanoprisms that sustain delocalized plasmon modes extending at the micrometer scale. Using TPL microscopy, we have imaged complex sub-particle intensity patterns that could be tuned by controlling the incident polarization or wavelength. Importantly, we have demonstrated formally that the TPL signal is directly related to the squared in-plane SP-LDOS, which echoes the well-established reciprocal relationship between emission lifetime and Ph-LDOS. However, SP-LDOS is more relevant to plasmonic information technology as it can be effectively engineered by controlling the shape or coupling such ultrathin prismatic single crystals into complex planar structures, which we propose to use as building blocks for plasmonic modal logic gates. Our results set a new paradigm of logic architecture design based on the direct engineering of the rich 2D SP-LDOS. This plasmonic technology extends the concept of integrated twodimensional devices recently proposed in monomolecular ${ }^{44}$ and graphene ${ }^{45}$ electronics to the optical processing of information. 


\section{Methods}

$\mathrm{Au}$ nanoprisms synthesis. The $\mathrm{Au}$ nanoprisms were prepared by increasing the $\mathrm{pH}$ of an aqueous solution containing $0.143 \mathrm{mM} \mathrm{NaNO}, 0.143 \mathrm{mM} \mathrm{KI}, 0.477 \mathrm{mM} \mathrm{HAuCl} 4$ and 19.1 mM PVP (total volume $209.6 \mathrm{~mL}$ ) by addition of $0.2 \mathrm{~mL}$ of $1 \mathrm{M} \mathrm{NaOH}$, diluted in water and left undisturbed for $18 \mathrm{~h}$. The Au nanoprisms were collected without any purification steps by decanting the supernatant and re-dispersing the colloids in deionized water. The nanoprisms suspension was drop-casted onto copper grids bearing a carbon film and washed with deionized water prior to structural characterization by transmission electron microscopy (TEM).

Sample preparation. $150 \mu \mathrm{m}$-thick glass substrates, coated with $10-20 \mathrm{~nm}$ of conductive Indium Tin Oxide (ITO), were purchased from DiamondCoatings Ltd. Labelled alignment cross-marks in aluminium were patterned every $200 \mu \mathrm{m}$ by optical lithography. The patterned substrates were thoroughly cleaned in successive ultrasonic baths of acetone and isopropanol. Oxygen RF plasma cleaning was performed for 5 min just before nanoprism deposition.

Gold nanoprisms were drop-casted for 5 min before immersing the substrate in a warm water bath for 2 min with gentle stirring. The final layers of PVP were removed by a 5 min oxygen plasma etch. The typical resulting surface density of the nanoprisms was about 0.15 particles per $1 \mu^{2}$. SEM imaging (Zeiss 1540XB) was performed in order to identify individual prisms to be studied and measure their lateral dimensions. SEM was again performed after TPL experiments to assess they retained morphology. AFM imaging performed on a Dimension 3000 Veeco-Bruker microscope in tapping mode provided the nanoprisms height after the entire preparation process and after the TPL experiments.

TPL microscopy. The linearly polarized beam of a Ti:Sapphire femtosecond laser (Coherent Chameleon Ultra II) delivering 120 fs near-infrared pulses tunable between 680 and $1080 \mathrm{~nm}$ was tightly focused onto the sample in a home-built optical microscope through a high numerical aperture oil immersion microscope objective (Olympus 100X, NA 1.35). The laser power used to collect enough two-photon luminescence (TPL) signal was kept in the 100$300 \mu \mathrm{W}$ range (as measured at the back aperture of the microscope objective), in order to avoid any heat-induced morphological changes of the nanoprisms. The polarization of the incident light was controlled by a $\lambda / 2$ plate (Thorlabs AHWP05M-980). The laser beam was modulated with an optical chopper at $6 \mathrm{kHz}$ to allow lock-in detection. TPL was collected in epi-collection geometry, separated from the excitation beam by a dichroic mirror (Semrock, FF665-Di02) and focused on a photomultiplier tube (Hamamatsu H7422P-40) operated in analog mode. Additional filters (Schott, BG39) were inserted at the entrance of the PMT. The 
detection path was carefully shielded from external stray light. The sample was mounted on a XY piezostage (Mad City Labs Nano-PDQ250) and raster scanned in the excitation focal spot with a typical scan step of 25- $50 \mathrm{~nm}$ in $\mathrm{X}$ and Y directions. The output of the lock-in amplifier (SRS 830) was acquired at every point of the map with a homemade LabView program. We observed that the TPL intensity varies quadratically with the incident power. The map patterns are stable in time, which excludes thermal or mechanical drifts, modification of the sample such as melting or displacement as confirmed a posteriori by further SEM and AFM analysis. Gaussian interpolation was applied to all maps except Figure $1 d$ which shows the raw data (See SI).

Numerical simulations. Our numerical tool is based on the three-dimensional Green dyadic method (3D-GDM) described in reference ${ }^{40}$. It allows the precise computation of the local electromagnetic field inside any arbitrary three-dimensional (3D) metal architectures placed on a substrate. The TPL signal emitted by the illuminated sample is computed from the local electric field distribution. In order to solve the local field distributions inside the metal particles, when they are excited by the three-dimensional optical light beam, the Green dyadic tensor inside the plasmonic structures is calculated first by discretizing the volume of the metallic objects with a hexagonal lattice of cells. In a second step, the integration of the squared local electric field intensity on the whole structure volume gives rise to the TPL signal expected for a particular position of the Gaussian beam waist center. Finally, by raster scanning the light beam on the sample, the data recording process is simulated for each focal spot location. In this manner, the TPL images are computed, pixel by pixel, through a complete self-consistent scheme including all the mutual interactions between the plasmonic platelets and the substrate. The model presented in the main text and detailed in the SI shows that TPL maps result from the convolution of the SP-LDOS distribution and the Gaussian profile of the probing beam. SP-LDOS maps can be conveniently calculated by using a circularly polarized optical beam with a waist reduced below the diffraction limit. 


\section{References}

1. Brongersma, M. L., Hartman, J. W. \& Atwater, H. A. Electromagnetic energy transfer and switching in nanoparticle chain arrays below the diffraction limit. Physical Review B 62, R16356-R16359 (2000).

2. Barnes, W. L., Dereux, A. \& Ebbesen, T. W. Surface plasmon subwavelength optics. Nature 424, 824-830 (2003).

3. Ozbay, E. Plasmonics: Merging photonics and electronics at nanoscale dimensions. Science 311, 189-193 (2006).

4. Pleros, N. et al. in IEEE Photonics Society, 2010 23rd Annual Meeting of the 165-166 (2010).

5. Bozhevolnyi, S. I., Volkov, V. S., Devaux, E., Laluet, J. Y. \& Ebbesen, T. W. Channel plasmon subwavelength waveguide components including interferometers and ring resonators. Nature 440, 508-511 (2006).

6. Grandidier, J. et al. Gain-Assisted Propagation in a Plasmonic Waveguide at Telecom Wavelength. Nano Letters 9, 2935-2939 (2009).

7. Drezet, A. et al. Leakage radiation microscopy of surface plasmon polaritons. Materials Science and Engineering: B 149, 220-229 (2008).

8. Krenn, J. R. et al. Squeezing the optical near-field zone by plasmon coupling of metallic nanoparticles. Physical Review Letters 82, 2590-2593 (1999).

9. Rang, M. et al. Optical Near-Field Mapping of Plasmonic Nanoprisms. Nano Letters 8, 3357-3363 (2008).

10. Tam, F., Goodrich, G. P., Johnson, B. R. \& Halas, N. J. Plasmonic enhancement of molecular fluorescence. Nano Letters 7, 496-501 (2007).

11. Kinkhabwala, A. et al. Large single-molecule fluorescence enhancements produced by a bowtie nanoantenna. Nature Photonics 3, 654-657 (2009).

12. Camden, J. P., Dieringer, J. A., Zhao, J. \& Van Duyne, R. P. Controlled Plasmonic Nanostructures for Surface-Enhanced Spectroscopy and Sensing. Accounts of Chemical Research 41, 1653-1661 (2008).

13. Chicanne, C. et al. Imaging the local density of states of optical corrals. Physical Review Letters 88, 097402 (2002).

14. Bouhelier, A. et al. Surface plasmon characteristics of tunable photoluminescence in single gold nanorods. Physical Review Letters 95, 267405 (2005).

15. Schuck, P. J., Fromm, D. P., Sundaramurthy, A., Kino, G. S. \& Moerner, W. E. Improving the mismatch between light and nanoscale objects with gold bowtie nanoantennas. Physical Review Letters 94, 017402 (2005).

16. Beversluis, M. R., Bouhelier, A. \& Novotny, L. Continuum generation from single gold nanostructures through near-field mediated intraband transitions. Physical Review B 68, 115433 (2003).

17. Ghenuche, P., Cherukulappurath, S., Taminiau, T. H., van Hulst, N. F. \& Quidant, R. Spectroscopic Mode Mapping of Resonant Plasmon Nanoantennas. Physical Review Letters 101, 116805 (2008).

18. McLeod, A. et al. Nonperturbative Visualization of Nanoscale Plasmonic Field Distributions via Photon Localization Microscopy. Physical Review Letters 106, 037402 (2011).

19. Vesseur, E. J. R., García de Abajo, F. J. \& Polman, A. Modal Decomposition of Surface-Plasmon Whispering Gallery Resonators. Nano Letters 9, 3147-3150 (2009).

20. Aeschlimann, M. et al. Adaptive subwavelength control of nano-optical fields. Nature 446, 301-304 (2007).

21. Douillard, L. et al. Short range plasmon resonators probed by photoemission electron microscopy. Nano Letters 8, 935-940 (2008). 
22. Nelayah, J. et al. Mapping surface plasmons on a single metallic nanoparticle. Nature Physics 3, 348-353 (2007).

23. Duan, H., Fernández-Domínguez, A. I., Bosman, M., Maier, S. A. \& Yang, J. K. W. Nanoplasmonics: Classical down to the Nanometer Scale. Nano Letters 12, 1683-1689 (2012).

24. Gu, L. et al. Resonant wedge-plasmon modes in single-crystalline gold nanoplatelets. Physical Review B 83, 195433 (2011).

25. Garcia de Abajo, F. J. \& Kociak, M. Probing the photonic local density of states with electron energy loss spectroscopy. Physical Review Letters 100, 106804 (2008).

26. Hoogenboom, J. P. et al. The Single Molecule Probe: Nanoscale Vectorial Mapping of Photonic Mode Density in a Metal Nanocavity. Nano Letters 9, 1189-1195 (2009).

27. Schuller, J. A. et al. Plasmonics for extreme light concentration and manipulation. Nature Materials 9, 193-204 (2010).

28. Wei, H., Wang, Z., Tian, X., Kall, M. \& Xu, H. Cascaded logic gates in nanophotonic plasmon networks. Nat Commun 2, 387 (2011).

29. Oulton, R. F. et al. Plasmon lasers at deep subwavelength scale. Nature 461, 629-632 (2009).

30. Dickson, R. M. \& Lyon, L. A. Unidirectional Plasmon Propagation in Metallic Nanowires. The Journal of Physical Chemistry B 104, 6095-6098 (2000).

31. Ditlbacher, H. et al. Silver Nanowires as Surface Plasmon Resonators. Physical Review Letters 95, 257403 (2005).

32. Huang, J. S. et al. Atomically flat single-crystalline gold nanostructures for plasmonic nanocircuitry. Nature Communications 1, 150 (2010).

33. Dorfmuller, J. et al. Fabry-Perot Resonances in One-Dimensional Plasmonic Nanostructures. Nano Letters 9, 2372-2377 (2009).

34. Pyayt, A. L., Wiley, B., Xia, Y. N., Chen, A. \& Dalton, L. Integration of photonic and silver nanowire plasmonic waveguides. Nature Nanotechnology 3, 660-665 (2008).

35. Song, M. X. et al. Imaging Symmetry-Selected Corner Plasmon Modes in PentaTwinned Crystalline Ag Nanowires. Acs Nano 5, 5874-5880 (2011).

36. The experimental fwhm spot diameter is estimated by the formula fwhm $=$ 0.515.lambda/NA, with a numerical apertue of $\mathrm{NA}=1.35$. The simulated beam waist value is approximated by lambda/2n, where $n=1.5$ is the glass refractive index.

37. Awada, C. et al. Selective Excitation of Plasmon Resonances of Single Au Triangles by Polarization-Dependent Light Excitation. Journal of Physical Chemistry C 116, 14591-14598 (2012).

38. Imura, K., Nagahara, T. \& Okamoto, H. Near-Field Two-Photon-Induced Photoluminescence from Single Gold Nanorods and Imaging of Plasmon Modes. The Journal of Physical Chemistry B 109, 13214-13220 (2005).

39. Boyd, G. T., Yu, Z. H. \& Shen, Y. R. Photoinduced luminescence from the noble metals and its enhancement on roughened surfaces. Physical Review B 33, 7923-7936 (1986).

40. Teulle, A. et al. Scanning optical microscopy modeling in nanoplasmonics. J. Opt. Soc. Am. B 29, 2431-2437 (2012).

41. Girard, C. et al. Imaging surface photonic states with a circularly polarized tip. Europhysics Letters 68, 797-803 (2004).

42. Fang, Y. R. et al. Branched Silver Nanowires as Controllable Plasmon Routers. Nano Letters 10, 1950-1954 (2010).

43. Shannon, C. E. A Symbolic Analysis of Relay and Switching Circuits. Transactions of the American Institute of Electrical Engineers 57, 713-723 (1938). 
44. Joachim, C., Gimzewski, J. K. \& Aviram, A. Electronics using hybrid-molecular and mono-molecular devices. Nature 408, 541-548 (2000).

45. Bao, Q. L. \& Loh, K. P. Graphene Photonics, Plasmonics, and Broadband Optoelectronic Devices. Acs Nano 6, 3677-3694 (2012). 


\section{Acknowledgements}

This work was supported by the European Research Council (ERC) (contract number ERC2007-StG Nr 203872 COMOSYEL), Agence Nationale de la Recherche (ANR) (Grant ANR09- BLAN-0049-01 Plastips), the CPER “Gaston Dupouy” 2007-2013 and the massively parallel computing center CALMIP in Toulouse.

\section{Author contributions}

E. D., A. A. and C. G. designed the research.

J. S. and E.D. developed the new synthesis of nanoprisms and J. S. produced the samples.

S. V., A. T. and E. D. prepared the TPL samples and performed the SEM and AFM imaging.

A. A. and R. M. developed the optical set-up. S. V., A. A., A. T. and R. M. performed the TPL experiments.

C. G. conceived the theoretical framework and implemented the simulation codes with R. M. and A. T. Simulations were performed by C. G., R. M. and A. T.

All authors contributed to the data analysis, figure preparation and manuscript writing.

Correspondence and requests for materials should be addressed to Arnaud Arbouet or Erik Dujardin.

\section{Competing financial interests}

The authors declare no competing financial interests. 

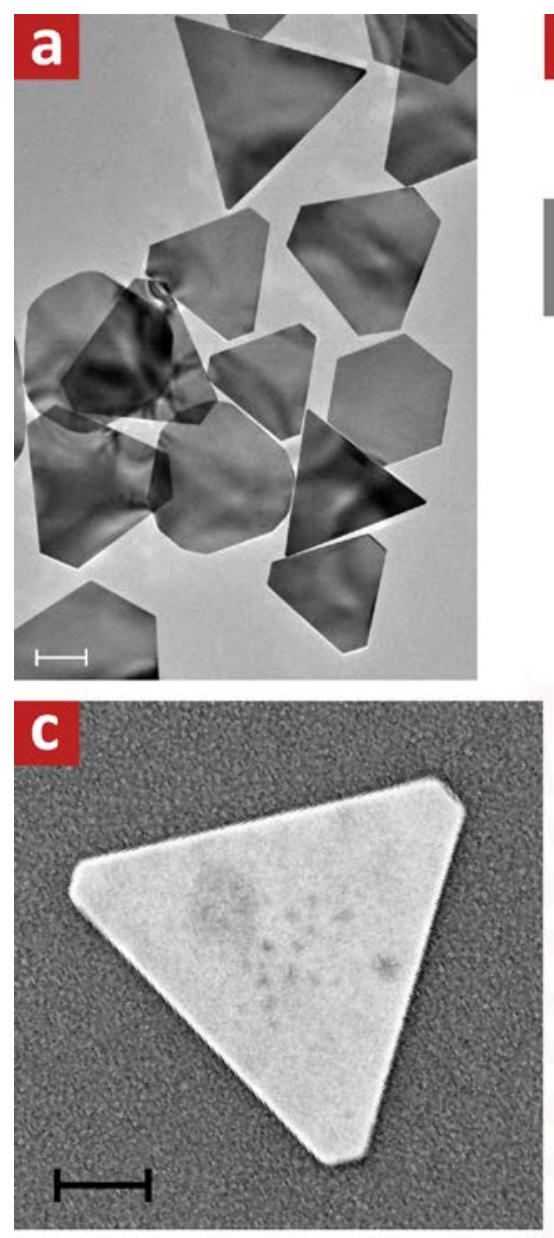
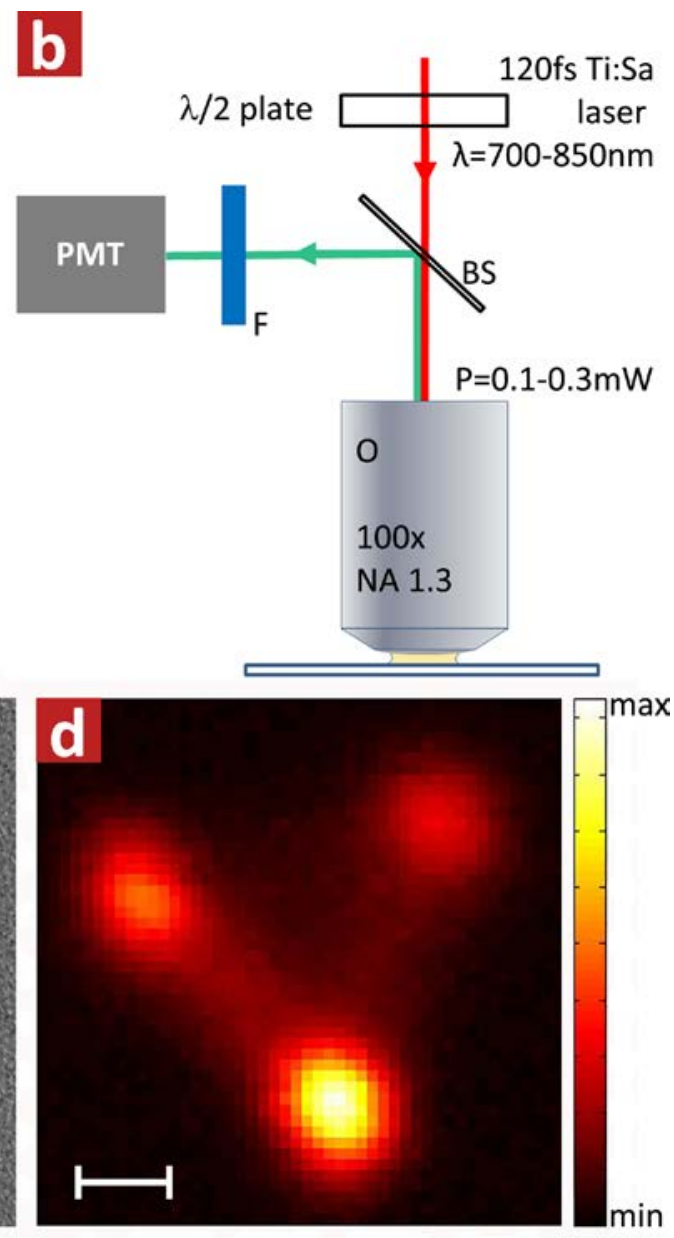

Figure 1. Crystalline Au nanoprisms and two-photon luminescence (TPL) microscopy. (a) Transmission electron microscopy image of the as-synthesized triangular, truncated triangular and hexagonal Au nanoprisms. (b) Schematics of the TPL set-up in epi-collection. The linearly polarized incident tunable pulsed Ti:Sa laser beam (pulse width $120 \mathrm{fs}$ ) is operated at $700-850 \mathrm{~nm}$. The direction of the polarization is controlled by a $\lambda / 2$ plate. The sample is illuminated through a NA 1.35 objective and the emitted light is collected in reflexion by a dichroic beam splitter (BS) and through a low pass filter (F) onto a photon multiplier tube (PMT). (c) Scanning electron micrograph of a specific nanoprism deposited on a glass / ITO substrate. (d) TPL image of the prism in (c) for an incident light set at $\lambda_{0}=700 \mathrm{~nm}$. The image is $50 \times 50$ pixels and displayed as raw data. Bars $=200 \mathrm{~nm}$. 


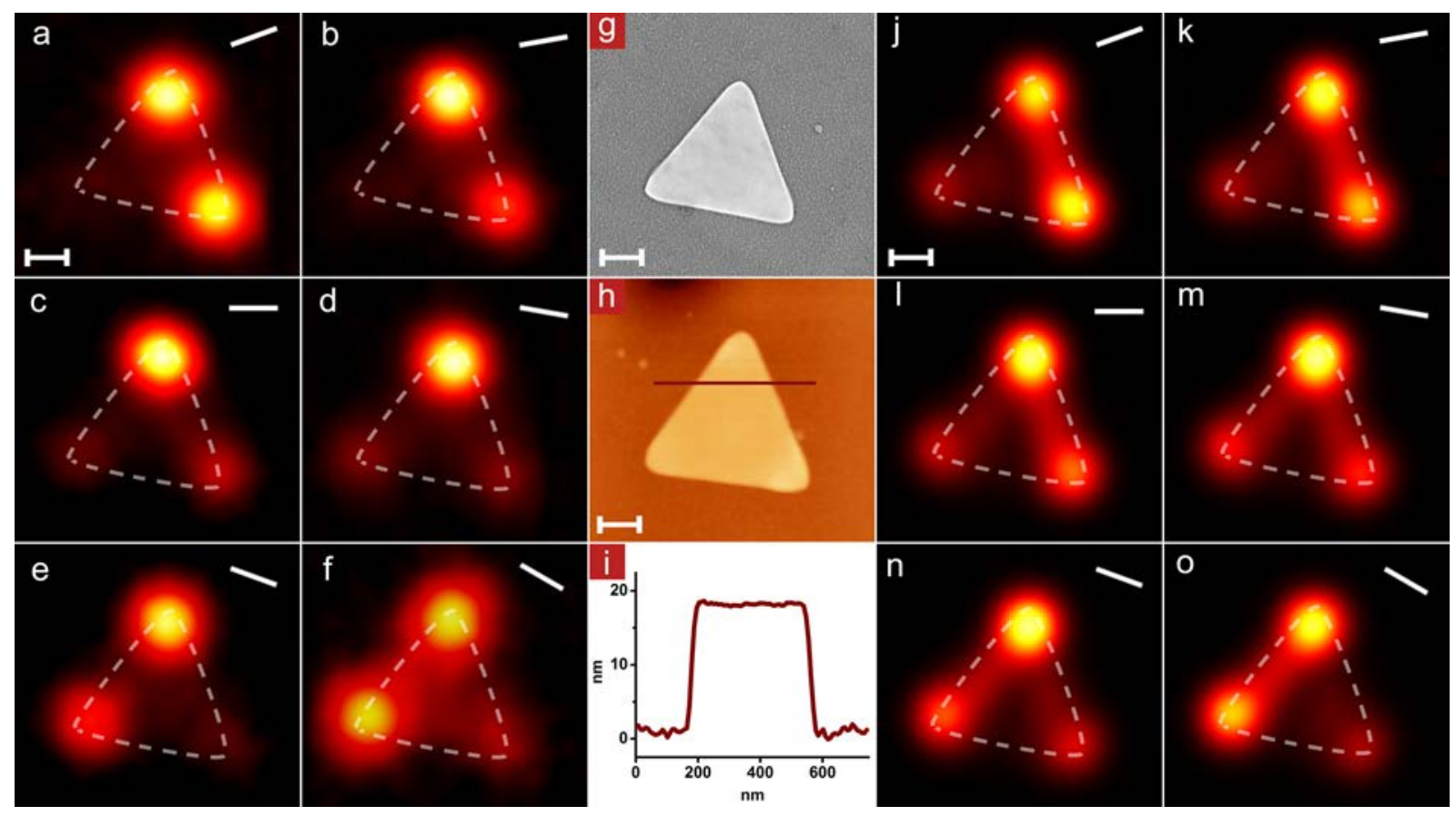

Figure 2. Polarization control of TPL maps. Experimental (a-f) and theoretical (j-o) TPL images of the $790 \mathrm{~nm}$ triangular nanoprism, observed by SEM micrograph in panel (g). (h) AFM image and (i) height profile of the same prism showing its uniform $20 \mathrm{~nm}$ thickness. The prism is excited at $\lambda_{0}=800 \mathrm{~nm}$ with a linearly polarized light. The direction of the inplane polarization is indicated in the upper right corners. In all images, the dotted white line is the outline of the considered nanoprism. Bars $=200 \mathrm{~nm}$. Color scale of $(a-f)$ and $(j-0)$ is similar to that of Fig. 1d. Simulation details for panels (j) to (o) are given in the main text and in Methods. Experimental TPL maps were processed as described in SI. 

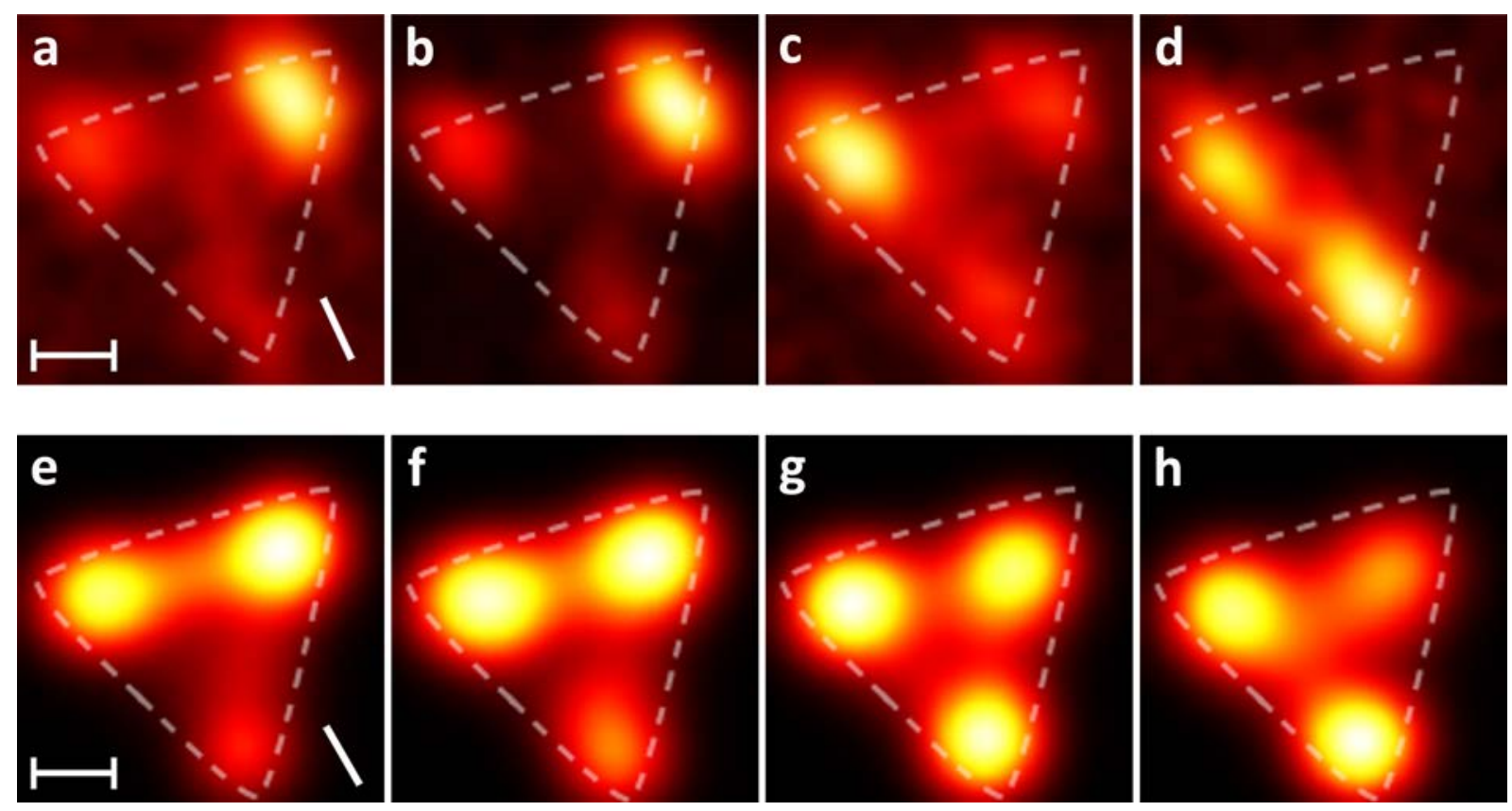

Figure 3. Influence of incident wavelength. (a-d) Experimental TPL images obtained on a 780-nm prism for a fixed incident linear polarization (shown in panel a) but for different $\lambda_{0}$ : (a) $700 \mathrm{~nm}$, (b) $750 \mathrm{~nm}$, (c) $800 \mathrm{~nm}$, (d) $850 \mathrm{~nm}$. (e-h) Simulated TPL maps taking into account the finite-sized gaussian beam waist of the incident light (diameter $300 \mathrm{~nm}$ ) for the same $\lambda_{0}$ : (e) $700 \mathrm{~nm}$, (f) $750 \mathrm{~nm}$, (g) $800 \mathrm{~nm}$, (h) $850 \mathrm{~nm}$. Bars = $200 \mathrm{~nm}$. Color scale is similar to that of Fig. 1d. Experimental TPL maps were processed as described in SI. 


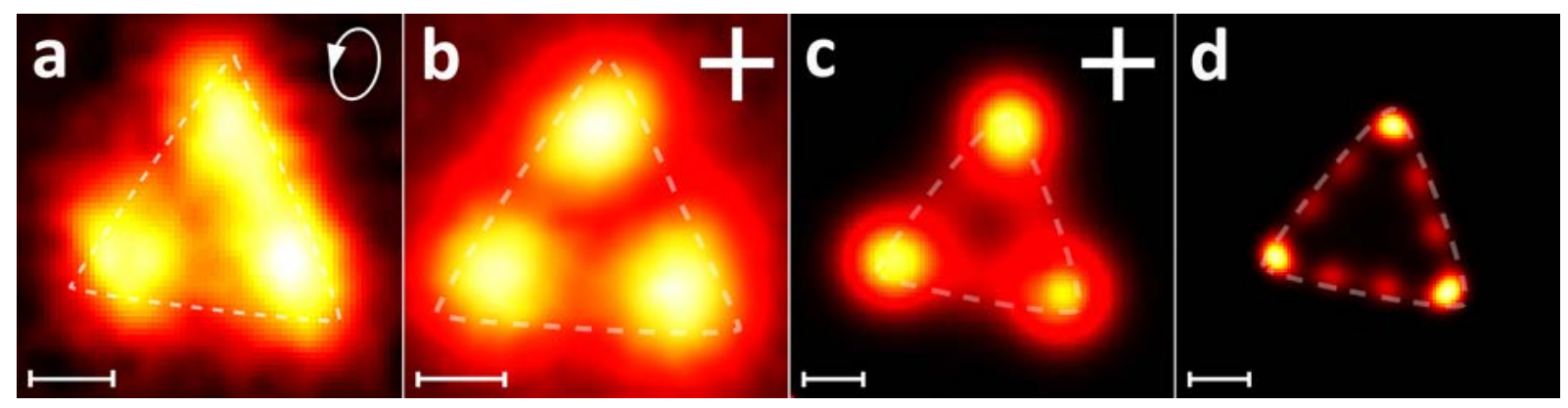

Figure 4. TPL signal and plasmonic density of states, SP-LDOS. (a) Experimental TPL map of an Au nanoprism excited with quasi-circularly polarized light. (b) Symmetrical TPL map of another nanoprism obtained by summing the images obtained for the two orthogonal linearly polarized excitation displayed in the upper right corner. (c) Simulated TPL map of panel (b) obtained with $\lambda_{0}=800 \mathrm{~nm}$ and a beam waist of $270 \mathrm{~nm}$. (d) Simulated TPL map identical to panel (c) but for a small beam waist (60 nm), which corresponds to the squared SP-LDOS. Bars $=200 \mathrm{~nm}$. Color scale is similar to that of Fig. 1d. Experimental TPL maps were processed as described in SI. 


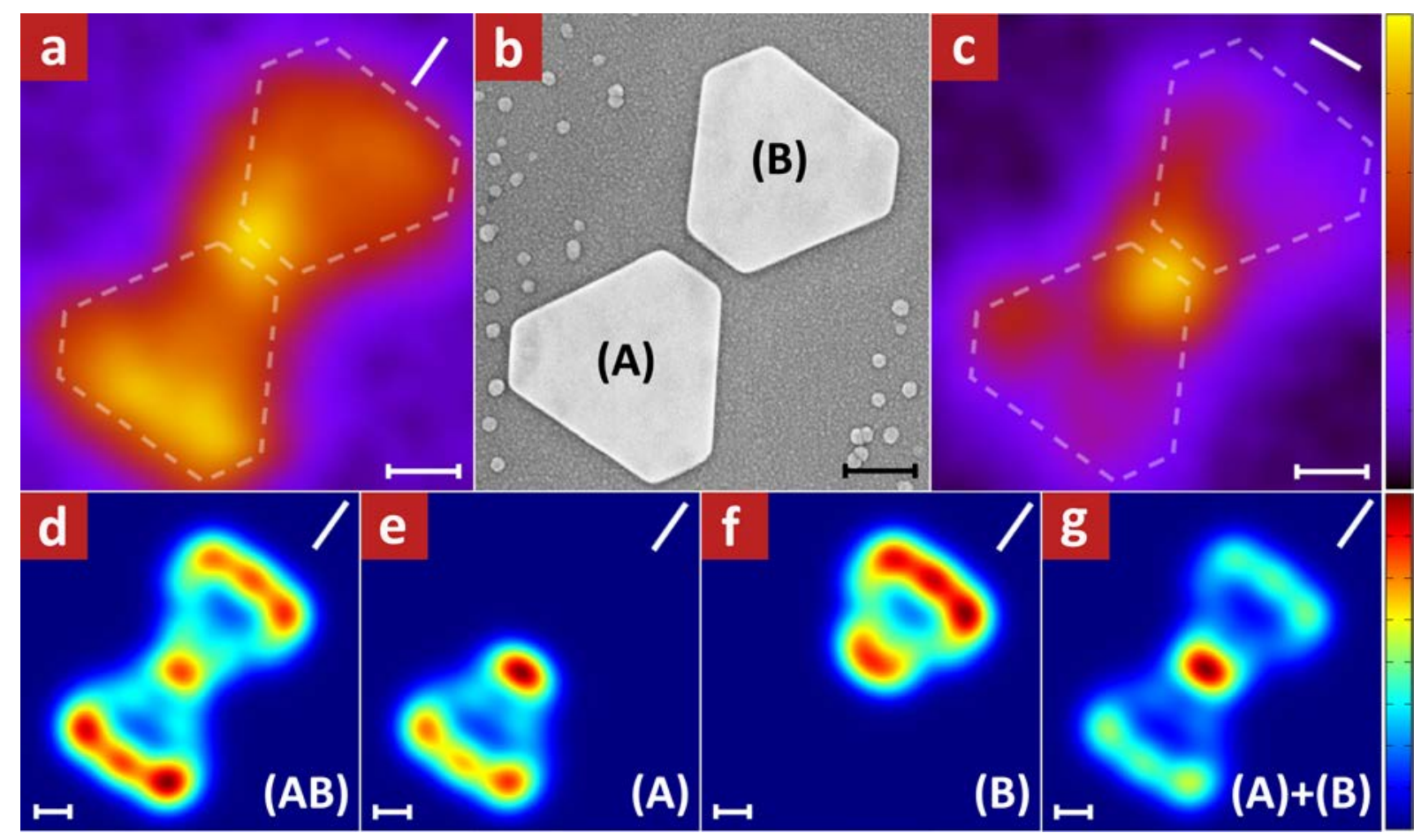

Figure 5. SP-LDOS engineering in coupled crystalline nanoprisms. (a) Experimental TPL map of the dimer of truncated triangular nanoprisms excited along the dimer longitudinal axis. (b) SEM image of the (A, B) dimer of prisms. (c) TPL map similar to panel (a) for an incident light polarized perpendicularly to the longitudinal axis of the dimer. (d) Simulated TPL map of the coupled dimer. (e, f) Simulated TPL maps of the isolated (A) and (B) nanoprisms. (g) TPL map resulting from the simple superposition of the signal $(e, f) . \lambda_{0}=800 \mathrm{~nm}$.

Bars $=200 \mathrm{~nm}$. Color scales for (a, c) and (d-g) are displayed on the side. Experimental TPL maps were processed as described in SI. 

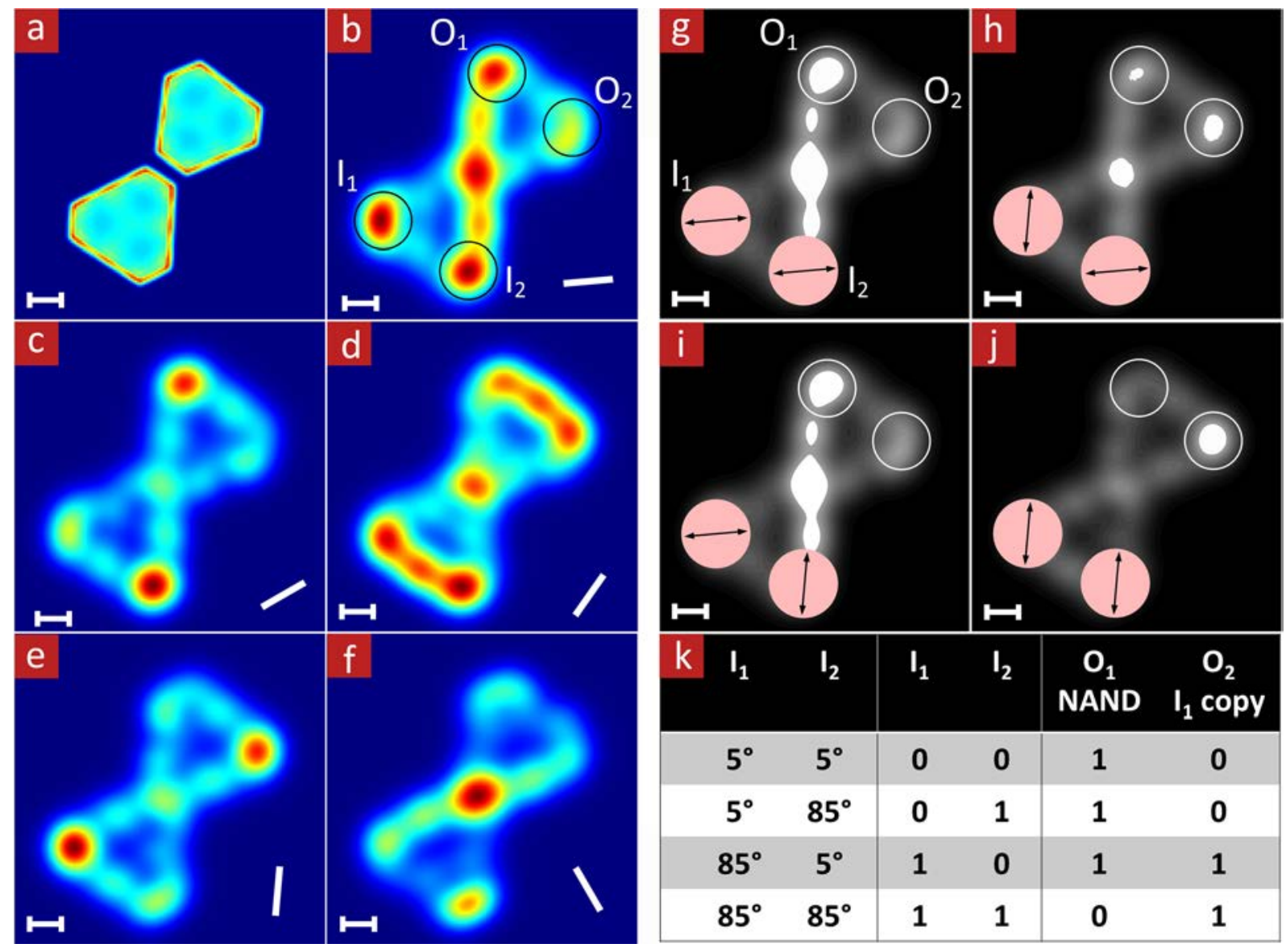

Figure 6. Plasmonic modal logic gates in coupled nanoprisms. (a) Simulated SP-LDOS map of the truncated triangular prism dimer shown in Figure 5b. (b-f) Simulated maps of the TPL signal for the coupled prisms for a series of incident linear polarization directions. (b) $5^{\circ}$, (c) $25^{\circ}$, (d) $55^{\circ}$, (e) $85^{\circ}$ (f) $120^{\circ} . \lambda_{0}=800 \mathrm{~nm}$, beam waist $=270 \mathrm{~nm}$. Color scale similar to Fig. 5(d-g). (g-j) Time-lapsed illumination of $I_{1}$ and $I_{2}$ with a polarization orientation of $5^{\circ}$ or $85^{\circ}$ is correlated to the time-integrated TPL signal in $\mathrm{O}_{1}$ and $\mathrm{O}_{2}$. The map (g) (resp. (i)) results from the sum of Fig. $6 b$ weighted by its maximal value in $I_{1}$ and Fig $6 b$ (resp. Fig. 6e) weighted by its maximal value in $\mathrm{I}_{2}$. It corresponds to inputs polarized at $5^{\circ}$ on $\mathrm{I}_{1}$ and $\mathrm{I}_{2}$ (resp. $5^{\circ}$ on $\mathrm{I}_{1}$ and $85^{\circ}$ on $\mathrm{I}_{2}$ ). Maps (j) (resp. (h)) are obtained by the same method. It corresponds to inputs polarized at $85^{\circ}$ on $\mathrm{I}_{1}$ and $\mathrm{I}_{2}$ (resp. $85^{\circ}$ on $\mathrm{I}_{1}$ and $5^{\circ}$ on $\mathrm{I}_{2}$ ). All four maps have been threshold to the same value. (k) Table summarizing the four illumination / detection configurations. Polarization of $5^{\circ}$ (resp. $85^{\circ}$ ) in $\mathrm{I}_{1}$ or $\mathrm{I}_{2}$ is ascribed a ' 0 ' (resp. '1') binary code. Intensity in $\mathrm{O}_{1}$ and $\mathrm{O}_{2}$ is ascribed a '0' (resp. '1') digital value if they are below (resp. above) the common threshold. $\mathrm{O}_{1}$ accomplishes a NAND gate while the $\mathrm{I}_{1}$ input is copied in $\mathrm{O}_{2}$. Bars $=200 \mathrm{~nm}$. 


\title{
Tailoring and imaging the plasmonic Local Density of States in crystalline nanoprisms
}

\author{
Sviatlana Viarbitskaya, Alexandre Teulle, Renaud Marty, Jadab Sharma, \\ Christian Girard, Arnaud Arbouet* and Erik Dujardin*
}

CEMES CNRS UPR 8011 and Université de Toulouse, 29 rue Jeanne Marvig, BP 94347, 31055 Toulouse cedex 4, France.

e-mail: arbouet@cemes.fr, dujardin@cemes.fr

\section{Contents}

1 Synthesis of single-twinned Au nanoprisms 1

2 TPL Image processing 2

3 Existing techniques for electromagnetic LDOS mapping 2

4 Relationship between TPL signal and SP-LDOS 3

4.1 Induced local electric field and TPL signal . . . . . . . . . . . . . . . 4

4.2 Optical probing of Surface Plasmon Local Density of States (SP-LDOS) . 5

4.3 Photonic LDOS computed above the plasmonic structure . . . . . . . . . 7

\section{Synthesis of single-twinned Au nanoprisms}

Single crystalline $\mathrm{Au}$ or Ag nanostructures produced by colloidal chemistry sustain high quality factor plasmonic modes thanks to their low dissipation [1]. The extension of single crystalline growth to larger sizes enables the investigation of the spatial distribution of electric fields and SP modes by simple optical microscopy on micrometric objects such as penta-twinned $\mathrm{Au}$ or $\mathrm{Ag}$ nanowires $[2,3,4]$. Yet atomically flat crystalline platelets are better suited for the observation of the two-dimensional (2D) extension of SP modes. We have produced $\mathrm{Au}$ nanoprisms with micrometric lateral sizes by a new one-pot method based on the direct reduction of Au precursors by polyvinylpyrrolidone (PVP) in alkaline conditions at room temperature (See Methods). The oxygen-rich PVP is known to trigger 
the anisotropic 2D growth by binding preferentially to $\{111\}$ crystallographic facets but, here, also acts as a reducing agent at elevated $\mathrm{pH}$, in contrast to classical methods that require either hydrothermal conditions or an additional reducing agent $[5,6]$. By modulating the reactivity of the gold precursor in the presence of halide ions and the $\{111\}$ growth inhibition by PVP and nitrate, the size of the prisms can be increased. Figure 1a shows that the sample is composed of $0.5-1.0 \mu \mathrm{m}$ equilateral triangular platelets with various degrees of truncation reaching the symmetrical hexagonal morphology. A detailed analysis, including electron diffraction and spherical aberration-corrected TEM, shows that the prisms are composed of two stacked single crystals sharing a twin boundary along the $\{111\}$ zone axis that extends laterally over the entire prisms (data not shown).

\section{TPL Image processing}

Two-Photon Luminescence (TPL) images are acquired by raster scanning a XY piezostage with typical scan steps of 25-50 nm. In Figure S1, the raw TPL images of a slightly truncated triangle (Fig. S1a) and of a quasi-hexagon (Fig. S1b) are shown in Figures S1c and S1d respectively. These TPL images exhibit a moderate pixelization effect that arises from the finite scanning step, $25 \mathrm{~nm}$ in the example of Fig. S1. In order to get smoother, more continuous image data, we have processed the raw TPL images by applying a Gaussian blur filter. Homogeneous images were obtained by adjusting the filter to a minimal radius of the Gaussian blur function. Figures S1e and S1f are the corresponding processed maps. After extensive processing tests, we found that this post-processing represents the raw data faithfully, without losing necessary details or introducing artifacts.

\section{Existing techniques for electromagnetic LDOS map- ping}

The strength of the interaction of a plasmonic nanostructure with any electromagnetic field depends on the number of optical excitations that it can support in the spectral range spanned by the incident field. The Electromagnetic Local Density of States (LDOS), $\rho\left(\mathbf{r}, \omega_{\mathbf{0}}\right)$, which gives the number of electromagnetic modes per unit energy at any location $\mathbf{r}$ therefore governs the relaxation of a quantum emitter or the energy losses of a swift electron beam in the vicinity of the nano-object. It has a central role and is a strong motivation for experimentalists, in the same way as the electronic density of states of solids for Scanning Tunneling Microscopy or Electron Energy Loss Spectroscopy (EELS) specialists.

Both photon and electron-based techniques have been used to map the LDOS. For planar geometries or systems having translational invariance along the direction of the electron beam, EELS provides direct access to the LDOS, the electron energy loss probability being proportional to the LDOS projected along the direction of electron path $[7,8]$. In cathodoluminescence experiments, an electron beam impinging on a nano-object creates 

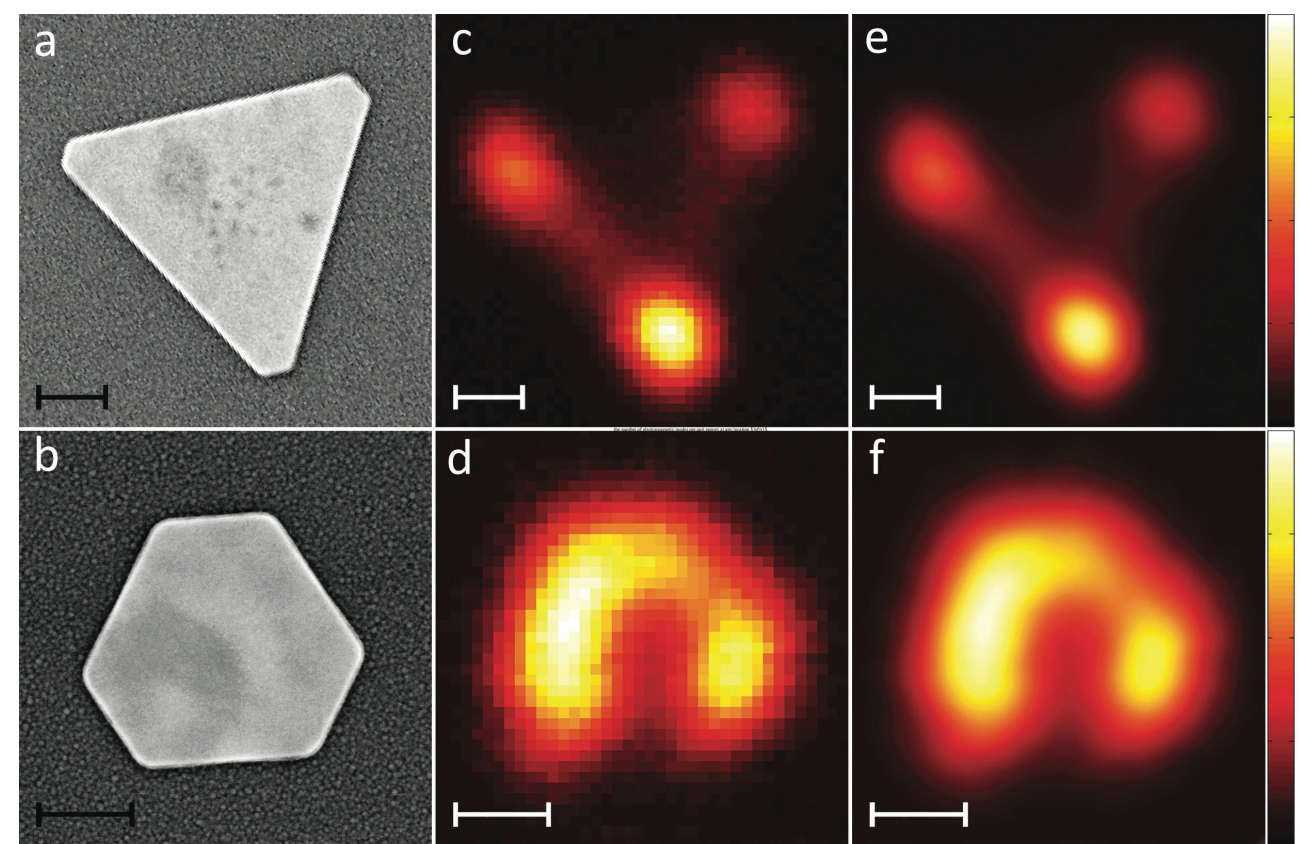

Figure S 1: Examples of the Gaussian blur filter applied to raw TPL maps. The as-acquired TPL maps of a truncated triangular (a) and a quasi-hexagonal (b) nanoprisms are shown in panels (c) and (d) respectively. The raw maps were acquired by raster scanning individual nanoprisms in the focal point of a linearly polarized $700 \mathrm{~nm}$ laser beam with a scanning step of $25 \mathrm{~nm}$. (e) and (f) are the processed images corresponding to the Gaussian Blur filtering of the images (c) and (d) respectively. Scales bars are $200 \mathrm{~nm}$.

a transient electric dipole at the impact location upon annihilation of its image charge. The corresponding radiated power is proportional to the partial LDOS along the direction of the transient dipole $[9,10]$. Scanning Near-field Optical Microscopy (SNOM) using a pointlike illuminating probe enables mapping of the LDOS projected along the direction of the effective dipole [11]. In this latter case, information about the LDOS is retrieved outside the nano-object at the tip apex location. Single molecules provide archetypal point-like probes of the LDOS. Their fluorescence decay rate is proportional to the partial LDOS along the direction of their transition dipole and Fluorescence Lifetime Imaging has been shown to spatially and vectorially map the LDOS by making use of single molecules as probes $[13,14]$.

\section{Relationship between TPL signal and SP-LDOS}

In the following, we show that despite its limited spatial resolution, far-field TPL microscopy gives access to the Local Density of States inside gold nanostructures. 


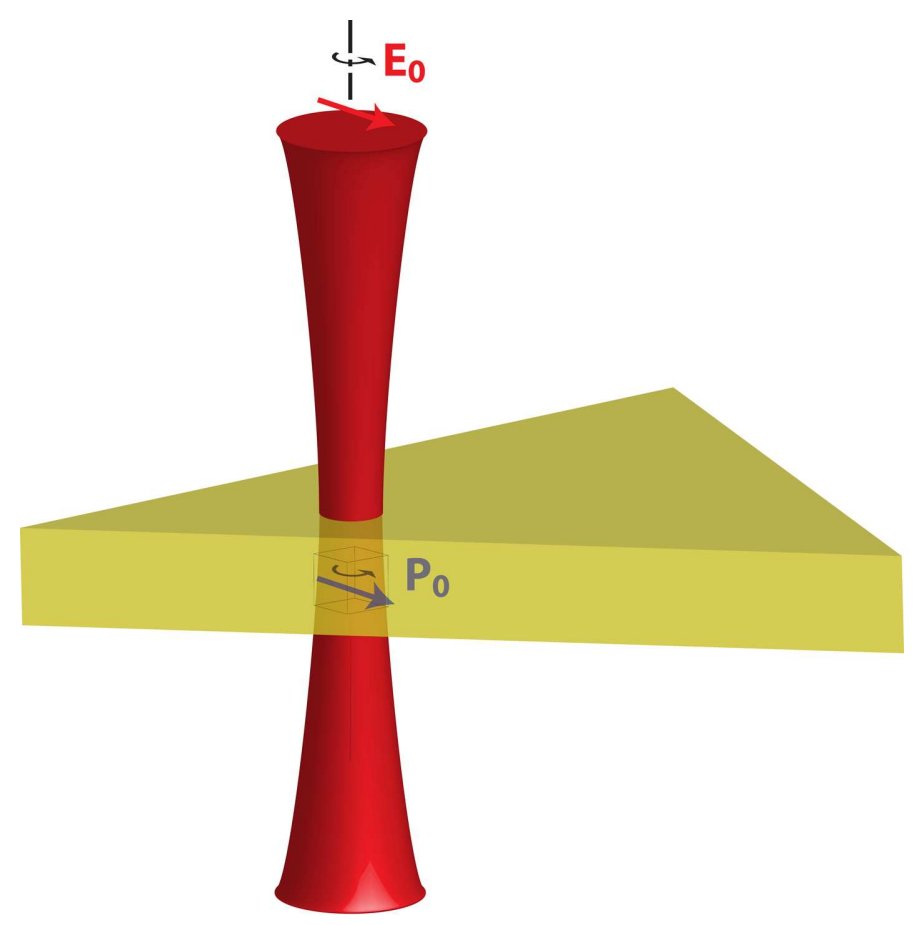

Figure S 2: Schematic geometry of an experimental configuration in which a focused light beam excites an individual metallic prism. $\mathbf{E}_{0}$ is the electric field of the incident light beam while the vector $\mathbf{P}_{0}$ is the dipole induced in the metal by $\mathbf{E}_{0}$. In the following, the beam center location will be defined by $\mathbf{R}_{0}$.

\subsection{Induced local electric field and TPL signal}

A numerical tool based on the Green dyadic method enables us to compute accurately the local electromagnetic distribution inside three-dimensional metal architectures with complex geometries lying or not on a substrate [15]. In the following, we apply this technique to the geometry depicted in Figure S2. Our approach is based on the numerical calculation of a unique generalized field propagator $\mathcal{K}\left(\mathbf{r}, \mathbf{r}^{\prime}, \omega_{0}\right)$ that contains the entire response of the sample to any incident field. Once $\mathcal{K}\left(\mathbf{r}, \mathbf{r}^{\prime}, \omega_{0}\right)$ has been computed, the response of the system to a given illumination field $\mathbf{E}_{\mathbf{0}}\left(\mathbf{R}_{0}, \mathbf{r}, \omega_{0}\right)$ is easily obtained by using

$$
\mathbf{E}\left(\mathbf{R}_{0}, \mathbf{r}, \omega_{0}\right)=\int_{V} \mathcal{K}\left(\mathbf{r}, \mathbf{r}^{\prime}, \omega_{0}\right) \cdot \mathbf{E}_{\mathbf{0}}\left(\mathbf{R}_{0}, \mathbf{r}^{\prime}, \omega_{0}\right) d \mathbf{r}^{\prime},
$$

where $\mathbf{E}\left(\mathbf{R}_{0}, \mathbf{r}, \omega_{0}\right)$ represents the local field inside or outside the plasmonic particle. This field distribution will change by varying the beam waist location $\mathbf{R}_{0}$. The integral in equation (1) runs over the volume $V$ occupied by the metallic particle [16].

TPL signal from gold involves second order nonlinear optical processes that can be described by introducing an effective $\omega$-dependent nonlinear coefficient $\eta\left(\omega_{0}\right)$ associated with the metal. From this coefficient and the local field distribution $\mathbf{E}\left(\mathbf{R}_{0}, \mathbf{r}_{i}, \omega_{0}\right)$ (cf. equation (1)) induced by the light beam inside the metal, we can write the TPL intensity 
generated by each elementary volume $v_{i}$ of the metal:

$$
\mathcal{I}_{i}\left(\mathbf{R}_{0}, \mathbf{r}_{i}, \omega_{0}\right)=\left[\eta\left(\omega_{0}\right)\left|\mathbf{E}\left(\mathbf{R}_{0}, \mathbf{r}_{i}, \omega_{0}\right)\right|^{2}\right]^{2} v_{i}
$$

In addition, as extensively described in relevant literature (see for example reference [17]), in gold TPL experiments the emission spectra appear as a broad background around twice the excitation frequency $\omega$. Consequently, it can be viewed as an incoherent process that can be described by adding the intensities emitted by the individual elementary cells:

$$
\mathcal{I}_{T P L}\left(\mathbf{R}_{0}, \omega_{0}\right)=\eta^{2}\left(\omega_{0}\right) \int_{V}\left|\mathbf{E}\left(\mathbf{R}_{0}, \mathbf{r}, \omega_{0}\right)\right|^{4} d \mathbf{r}
$$

As discussed in reference [16], this equation can be used to simulate TPL images of colloidal particles randomly distributed on a surface. When working with two-dimensional (2D) plasmonic building blocks, we will show, in the following section, that equation (3) can also be rewritten from the local density of states associated with the surface plasmons of the metal particle.

\subsection{Optical probing of Surface Plasmon Local Density of States (SP-LDOS)}

To start the demonstration, we consider the standard relation

$$
\mathcal{I}=A \chi^{2}\left(\omega_{0}\right)\left|\mathbf{E}\left(\mathbf{R}_{0}, \mathbf{r}, \omega_{0}\right) \delta \mathbf{r}\right|^{2}
$$

which gives the amount of energy radiated, during a linear process, by an elementary cell of volume $\delta \mathbf{r}$ located inside the metal (where $A$ is a real constant and $\chi\left(\omega_{0}\right)$ represents the linear susceptibility of the metal). This energy can be written in another equivalent manner by applying the dynamical Gauss' theorem to an induced dipole moment $\mathbf{P}_{0}\left(\mathbf{r}, \omega_{0}\right)$ $=\delta \mathbf{r} \chi\left(\omega_{0}\right) \mathbf{E}\left(\mathbf{R}_{0}, \mathbf{r}, \omega_{0}\right)$ located inside the metal (the complete demonstration is provided in reference [18]). Furthermore, if we consider that the source dipole of magnitude $\mathbf{P}_{0}$ is driven by an external monochromatic excitation of angular frequency $\omega_{0}$ and circularly polarized in a plane parallel to the mean surface sample (plane $X O Y$ ), as described in figure $\mathrm{S} 2$, we can write [18]:

$$
\mathcal{I}=P_{0}^{2} \pi^{2} \omega_{0}^{2} \rho_{\|}\left(\mathbf{r}, \omega_{0}\right),
$$

where $\rho_{\|}\left(\mathbf{r}, \omega_{0}\right)$ represents the SP-LDOS of the planar plasmonic structure:

$$
\rho_{\|}\left(\mathbf{r}, \omega_{0}\right)=\frac{1}{2 \pi^{2} \omega_{0}} \Im\left\{S_{x, x}\left(\mathbf{r}, \mathbf{r}, \omega_{0}\right)+S_{y, y}\left(\mathbf{r}, \mathbf{r}, \omega_{0}\right)\right\},
$$

in which the symbol $\Im$ means that the imaginary part of the quantity between brackets should be taken. Moreover, $S_{x, x}\left(\mathbf{r}, \mathbf{r}, \omega_{0}\right)$ and $S_{y, y}\left(\mathbf{r}, \mathbf{r}, \omega_{0}\right)$ are the first two diagonal components of the Green dyadic tensor $S\left(\mathbf{r}, \mathbf{r}, \omega_{0}\right)$ calculated at a point $\mathbf{r}$ located inside the metallic particle. 
After identification of equations (4) and (5), we get a general relation between light field intensity $\left|\mathbf{E}_{0}\left(\mathbf{R}_{0}, \mathbf{r}, \omega\right)\right|^{2}$, local field intensity $\left|\mathbf{E}\left(\mathbf{R}_{0}, \mathbf{r}, \omega\right)\right|^{2}$, and SP-LDOS at any arbitrary point $\mathbf{r}$ inside the metal:

$$
\left|\mathbf{E}\left(\mathbf{R}_{0}, \mathbf{r}, \omega\right)\right|^{2}=\left|\mathbf{E}_{0}\left(\mathbf{R}_{0}, \mathbf{r}, \omega\right)\right|^{2} \pi^{2} \omega_{0}^{2} A^{-1} \rho_{\|}\left(\mathbf{r}, \omega_{0}\right) .
$$

This equation shows in a concise way how the field intensity expected at a given point $\mathbf{r}$ inside the sample is governed by the density of plasmon states existing at the same point r. In other words, this relationship shows that it is not possible to induce a significant local field in a place where the density of states of plasmons is too low. Finally, using this general relation in the case of the localized TPL signal described by equation (3), leads to:

$$
\mathcal{I}_{T P L}\left(\mathbf{R}_{0}, \omega_{0}\right)=\eta^{2}\left(\omega_{0}\right) \pi^{4} \omega_{0}^{4} A^{-2} \int_{V}\left|\mathbf{E}_{0}\left(\mathbf{R}_{0}, \mathbf{r}, \omega\right)\right|^{4} \rho_{\|}^{2}\left(\mathbf{r}, \omega_{0}\right) d \mathbf{r}
$$

This equation provides the relationship between the TPL signal, recorded by scanning a tightly focused circularly polarized light beam, and the SP-LDOS map generated by the particle. Equation (8) clearly shows that the TPL intensity results from the convolution of the squared SP-LDOS, $\rho_{\|}^{2}\left(\mathbf{r}, \omega_{0}\right)$, with the gaussian profile of the light beam. Consequently, the TPL map features depend on the lateral size of the beam waist but do converge towards the SP-LDOS map when the beam waist is theoretically contracted to zero. The effect of

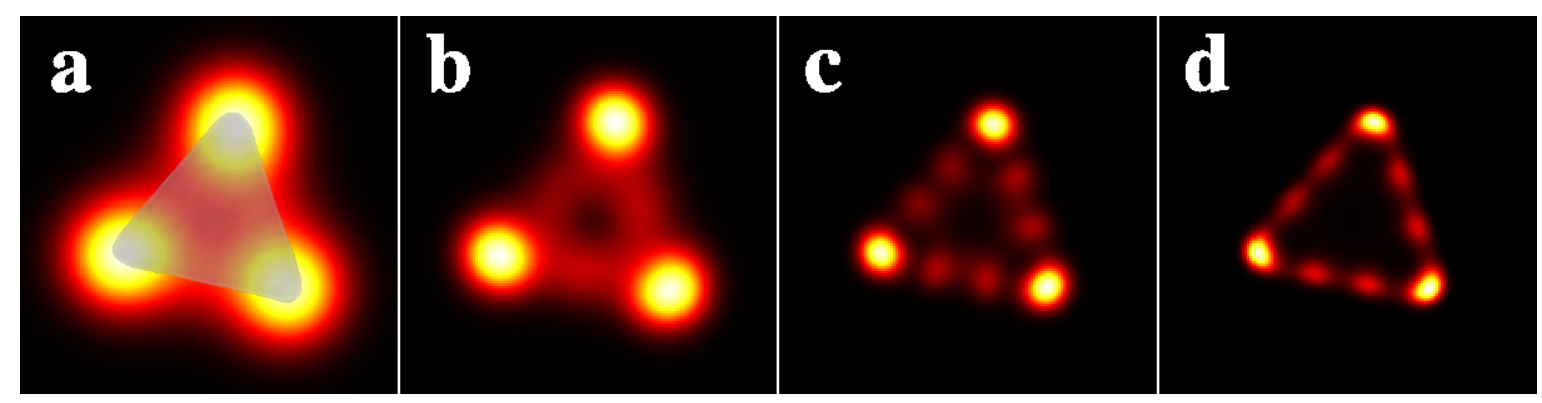

Figure S 3: Sequence of TPL maps computed with a gold triangular nanoprism illuminated with a circularly polarized light of decreasing beam waist diameter $(300 \mathrm{~nm}, 200 \mathrm{~nm}$, $100 \mathrm{~nm}$, and $50 \mathrm{~nm}$ for (a), (b), (c), (d)) respectively. A SEM image of the object is superimposed to the computed map in (a). Image size: $1.2 \mu \mathrm{m} \times 1.2 \mu \mathrm{m}$.

convolution is illustrated in Figure S3 that shows four different TPL intensity maps of the same triangular structure computed with beam waists of decreasing sizes.

Finally, when the light beam is linearly polarized along, for instance, the $O X$ axis, a relation similar to $(8)$ can be derived:

$$
\mathcal{I}_{T P L}\left(\mathbf{R}_{0}, \omega_{0}\right)=\eta^{2}\left(\omega_{0}\right) \pi^{4} \omega_{0}^{4} A^{-2} \int_{V}\left|\mathbf{E}_{0}\left(\mathbf{R}_{0}, \mathbf{r}, \omega\right)\right|^{4} \rho_{x}^{2}\left(\mathbf{r}, \omega_{0}\right) d \mathbf{r},
$$

in which the TPL signal is not directly proportional to $\rho_{\|}(\mathbf{r}, \omega)$, but provides information on the partial SP-LDOS (also called projected SP-LDOS) $\rho_{x}(\mathbf{r}, \omega)$ in the plane of the sample. 


\subsection{Photonic LDOS computed above the plasmonic structure}

For the sake of completeness, we present in figure S4 (a-c) a sequence of three Photonic LDOS (Ph-LDOS) maps computed above the single gold prism of Figure S3. The first image (Fig. S4a) corresponds to the mesoscopic range with a computation performed in the plane $\mathrm{Z}=100 \mathrm{~nm}$ parallel to the surface structure. At such distance, the $\mathrm{Ph}$-LDOS is surrounded by a complex ripple pattern that reveals the first non-evanescent photonic states lying around the particle. By decreasing the observation distance $\mathrm{Z}$ in the two following maps Fig. S4b and Fig. S4c, we observe a significant contraction of the PhLDOS pattern indicating that we enter the subwavelength range in which a significant increase of the evanescent states appears on the prism edges.

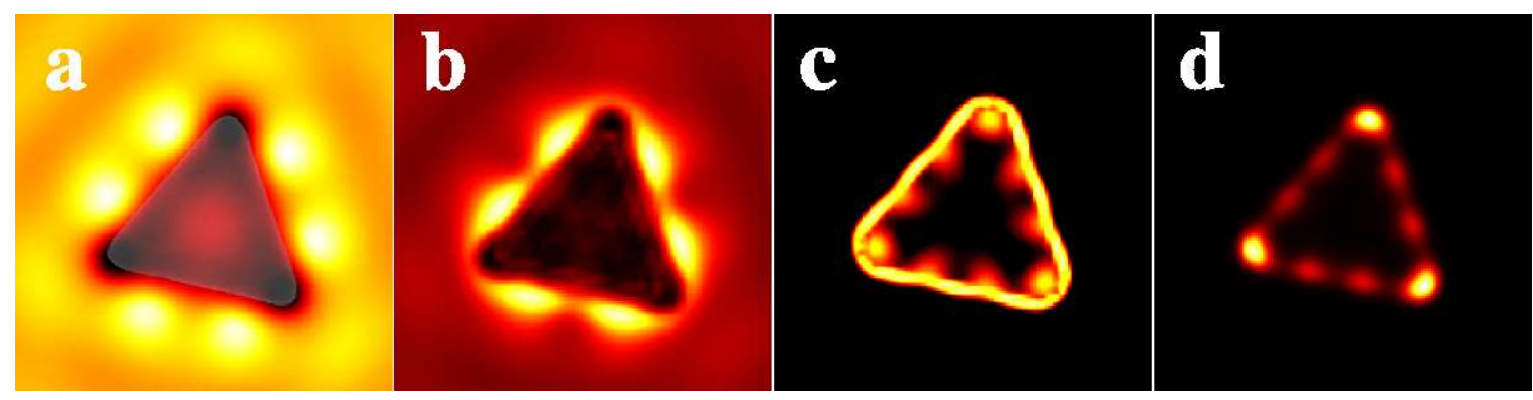

Figure S 4: (a), (b) and (c) photonic LDOS maps calculated in three planes $(\mathrm{Z}=$ constant) located at $Z=100 \mathrm{~nm}, Z=50 \mathrm{~nm}$, and $Z=25 \mathrm{~nm}$ above the plasmonic prism of Figure S3, respectively. (d) SP-LDOS of the same structure given for comparison. All physical parameters are the same as in Figure S3.

As expected, the last Ph-LDOS map Fig. S4c computed in the immediate vicinity of the metal ressembles the SP-LDOS computed inside the metal given in Fig. S4d. Nevertheless, images from Fig. S4c and Fig. S4d describe two different quantities related to the photonic and surface plasmon density of states, respectively. This comparison clearly indicates the interest of the TPL technique to provide a direct access to the SP-LDOS supported by metallic structures.

\section{References}

[1] Ditlbacher, H. et al, Phys. Rev. Let. 95, 257403, (2005)

[2] Dickson, R. M. and Lyon, L. A., J. Phys. Chem. B 104, 6095-6098, (2000)

[3] Pyayt, A. L., Wiley, B., Xia, Y. N., Chen, A. and Dalton, L., Nature Nanotechnology 3, 660-665, (2008)

[4] Song, M. X. et al., Acs Nano 5, 5874-5880, (2011)

[5] Sun, Y. G. and Xia, Y. N., Science 298, 2176-2179, (2002) 
[6] Pastoriza-Santos, I. and Liz-Marzan, L. M., Langmuir 18, 2888-2894, (2002)

[7] Garcia de Abajo, F. J., Rev. Mod. Phys., 82, 209, (2010)

[8] Garcia de Abajo, F. J. and Kociak, M., Phys. Rev. Lett., 100, 106804, (2008)

[9] Sapienza, R.; Coenen, T.; Renger, J.; Kuttge, M.; van Hulst, N. F. and Polman, A., Nature Materials, 11, 781-787, (2012)

[10] Kuttge, M.; Vesseur, E. J. R.; Koenderink, A. F.; Lezec, H. J.; Atwater, H. A.; Garcia de Abajo, F. J. and Polman, A., Phys. Rev. B, 79, 113405, (2009)

[11] Colas des Francs, G.; Girard, C.; Weeber, J.-C. and Dereux, A. , Chemical Physics Letters, 345, 512-516, (2001)

[12] Douillard, L. and Charra, F., Comptes Rendus Physique, 13, 815-829, (2012)

[13] Michaelis, J.; Hettich, C.; Mlynek, J. and Sandoghdar, V., Nature 405, 325-328, 2000

[14] Hoogenboom, J. P.; Sanchez-Mosteiro, G.; des Francs, G. C.; Heinis, D.; Legay, G.; Dereux, A. and van Hulst, N. F., Nano Letters, 9, 1189-1195, (2009)

[15] C. Girard, E. Dujardin, G. Baffou, R. Quidant, New Journal of Physics, 10, 105016 (2008).

[16] A. Teulle, R. Marty, S. Viarbitskaya, A. Arbouet, E. Dujardin, C. Girard, and G. Colas des Francs, J. Opt. Soc. Am., 29, 2431 (2012).

[17] H. Okamoto and K. Imura, Progress in Surface Science, 84, 199-229 (2009).

[18] C. Girard, T. David, C. Chicanne, A. Mary, G. Colas des Francs, E. Bourillot, J -C. Weeber, and A. Dereux, Europhysics Letters, 68, 797 (2004). 